\title{
An Integral Containing the Square of a Bessel Function
}

\section{By J. N. Newman and W. Frank}

In the process of investigating the hydrodynamical characteristics of floating and submerged ellipsoids, an integral arose of the form

$$
I_{n}^{m}(x)=\int_{0}^{x / 2} \frac{J_{n}^{2}(x \cos \theta)}{(x \cos \theta)^{2 m}} d \theta
$$

where $m$ and $n$ are either integers, or integers plus one half, $0 \leqq m \leqq n$, and $J_{n}$ is the Bessel function of the first kind of order $n$. For the case where $m$ and $n$ are integers plus one half, the integral (1) can be conveniently expressed in terms of the spherical Bessel function $j_{n}(z)=\sqrt{\frac{\pi}{2 z}} J_{n+1 / 2}(z)$. Then

$$
I_{n+1 / 2}^{m+1 / 2}(x)=\frac{2}{\pi} \int_{0}^{\pi / 2} \frac{j_{n}{ }^{2}(x \cos \theta)}{(x \cos \theta)^{2 m}} d \theta .
$$

Since no reference could be found to the properties of the above integrals, we present here certain of their properties and a short table of numerical values. A more extensive table for integer values of $m$ and $n$, giving values to $5 \mathrm{~S}$, for $x=$ $.1(.1) 10.0, n=0(1) 9$, and $m=0(1) n$, is on deposit in the Unpublished Mathematical Tables file.

The integral (1) can be expressed as a generalized hypergeometric function ${ }_{2} F_{3}$ by using the Neumann series [1] for $J_{n}{ }^{2}$ and interchanging the orders of summation and integration. In this manner we obtain

$$
\begin{aligned}
& I_{n}{ }^{m}(x)=\frac{1}{2} \sum_{K=0}^{\infty} \frac{(-1)^{k} x^{2(K+n-m)} \Gamma\left(n+K+\frac{1}{2}\right) \Gamma\left(n+K-m+\frac{1}{2}\right)}{K ! \Gamma(n+K+1) \Gamma(2 n+K+1) \Gamma(K+n-m+1)} \\
&= \frac{\sqrt{\pi}}{2^{2 n+1}} \frac{\Gamma\left(n-m+\frac{1}{2}\right)}{[\Gamma(n+1)]^{2} \Gamma(n-m+1)} x^{2 n-2 m} \\
&{ }_{2} F_{3}\left(\begin{array}{c}
n+\frac{1}{2}, n-m+\frac{1}{2} ;-x^{2} \\
n+1,2 n+1, n-m+1
\end{array}\right)
\end{aligned}
$$

This series expansion is everywhere convergent and provides an efficient means for computing decimal approximations to the integrals (1) unless $x$ is very large. For large $x$, asymptotic representations can be derived as follows. Changing the variable of integration to $y=x \cos \theta$, it follows that

$$
\begin{aligned}
I_{n}{ }^{m}(x) & =\int_{0}^{x} \frac{J_{n}^{2}(y)}{y^{2 m} \sqrt{x^{2}-y^{2}}} d y \\
& =\int_{0}^{x^{1 / 2}} \frac{J_{n}^{2}(y)}{y^{2 m} \sqrt{x^{2}-y^{2}}} d y+\int_{x^{1 / 2}}^{x} \frac{J_{n}^{2}(y)}{y^{2 m} \sqrt{x^{2}-y^{2}}} d y .
\end{aligned}
$$

Received April 20, 1962. 
For $m \neq 0$ the contribution from the first interval is

$$
\begin{aligned}
\int_{0}^{x^{1 / 2}} \frac{J_{n}^{2}(y)}{y^{2 m} \sqrt{x^{2}-y^{2}}} d y & \cong \frac{1}{x} \int_{0}^{x^{1 / 2}} \frac{J_{n}^{2}(y)}{y^{2 m}} d y \\
& \cong \frac{1}{x} \int_{0}^{\infty} \frac{J_{n}^{2}(y)}{y^{2 m}} d y=\frac{1}{x} \frac{\Gamma(m) \Gamma\left(n-m+\frac{1}{2}\right)}{2 \sqrt{\pi} \Gamma\left(m+\frac{1}{2}\right) \Gamma\left(n+m+\frac{1}{2}\right)}
\end{aligned}
$$

and the contribution from the second interval is small, ${ }^{*}$ of order $x^{-(m+1)} \log x^{\text {. }}$ For $m=0$, the contribution from the first interval is

$$
\begin{aligned}
\int_{0}^{x^{1 / 2}} \frac{J_{n}{ }^{2}(y)}{\sqrt{x^{2}-y^{2}}} d y \cong \frac{1}{x} \int_{0}^{x^{1 / 2}} & J_{n}{ }^{2}(y) d y \\
& =\frac{1}{x} \int_{0}^{x^{1 / 2}}\left[J_{n}{ }^{2}(y)-f(y)\right] d y+\frac{1}{x} \int_{0}^{x^{1 / 2}} f(y) d y
\end{aligned}
$$

where $f(y)$ is arbitrary. The asymptotic behavior of ${J_{n}}^{2}(y)$ suggests setting

$$
f(y)=\frac{1}{\pi(y+1)},
$$

for, with such a choice,

$$
\begin{aligned}
& \int_{0}^{x^{1 / 2}}\left[{J_{n}}^{2}(y)-f(y)\right] d y \cong \int_{0}^{\infty}\left[J_{n}{ }^{2}(y)-f(y)\right] d y \\
& =\lim _{p \rightarrow 0} \int_{0}^{\infty} e^{-p y}\left[J_{n}^{2}(y)-f(y)\right] d y \\
& =\frac{1}{\pi} \lim _{p \rightarrow 0}\left[Q_{n-1 / 2}\left(1+\frac{1}{2} p^{2}\right)+e^{p} E i(-p)\right] \\
& =\frac{1}{\pi} \lim _{p \rightarrow 0}\left[-\gamma-\psi\left(n+\frac{1}{2}\right)-\log \frac{1}{2} p+\gamma+\log p\right] \\
& =\frac{1}{\pi}\left[\log 2-\psi\left(n+\frac{1}{2}\right)\right] .
\end{aligned}
$$

Here $Q_{n-1 / 2}$ is the Legendre function of the second kind, $E i(-p)$ is the exponential integral, and $\psi$ is the logarithmic derivative of the gamma function. Since

$$
\int_{0}^{x^{1 / 2}} f(y) d y=\frac{1}{\pi} \log \left(1+x^{1 / 2}\right)=\frac{1}{2 \pi} \log x+0\left(x^{-1 / 2}\right),
$$

it follows that

$$
\int_{0}^{x^{1 / 2}} \frac{J_{n}^{2}(y)}{\sqrt{x^{2}-y^{2}}} d y=\frac{1}{\pi x}\left[\log 2 x^{1 / 2}-\psi\left(n+\frac{1}{2}\right)\right]+0\left(x^{-3 / 2}\right) .
$$

* This follows by substituting the asymptotic expansion of $J_{n}$ for large argument and intergrating term-by-term. 
TABLE 1

The Integrals $I_{n}{ }^{m}(x)$

\begin{tabular}{|c|c|c|c|c|c|}
\hline$x$ & $I_{0}{ }^{0}(x)$ & $I_{1}{ }^{0}(x)$ & $I_{1}{ }^{1}(x)$ & $I_{2}{ }^{0}(x)$ & $I_{2}{ }^{1}(x)$ \\
\hline 0.0 & $0.15708 \mathrm{E} \quad 01$ & 0.00000 & $0.39270 \mathrm{E}-00$ & 0.00000 & 0.00000 \\
\hline 0.2 & $0.15552 \mathrm{E} \quad 01$ & $0.77953 \mathrm{E}-02$ & $0.39074 \mathrm{E}-00$ & $0.14645 \mathrm{E}-04$ & $0.48843 \mathrm{E}-03$ \\
\hline 0.4 & $0.15094 \mathrm{E} \quad 01$ & $0.30486 \mathrm{E}-01$ & $0.38494 \bar{E}-00$ & $0.23044 \mathrm{E}-03$ & $0.19246 \mathrm{E}-02$ \\
\hline 0.6 & $0.14364 \mathrm{E} \quad 01$ & $0.66061 \mathrm{E}-01$ & $0.37552 \mathrm{E}-00$ & $0.11345 \mathrm{E}-02$ & $0.42234 \mathrm{E}-02$ \\
\hline 0.8 & $0.13410 \mathrm{E} \quad 01$ & $0.11140 \mathrm{E}-00$ & $0.36281 \mathrm{E}-00$ & $0.34482 \mathrm{E}-02$ & $0.72495 \mathrm{E}-02$ \\
\hline 1.0 & $0.12293 \mathrm{E} \quad 01$ & $0.16257 \mathrm{E}-00$ & $0.34727 \mathrm{E}-00$ & $0.80041 \mathrm{E}-02$ & $0.10828 \mathrm{E}-01$ \\
\hline 1.2 & $0.11079 \mathrm{E} \quad 01$ & $0.21528 \mathrm{E}-00$ & $0.32943 \mathrm{E}-00$ & $0.15600 \mathrm{E}-01$ & $0.14754 \mathrm{E}-01$ \\
\hline 1.4 & $0.98399 \mathrm{E} \quad 00$ & $0.26522 \mathrm{E}-00$ & $0.30990 \mathrm{E}-00$ & $0.26850 \mathrm{E}-01$ & $0.18813 \mathrm{E}-01$ \\
\hline 1.6 & $0.86398 \mathrm{E} \quad 00$ & $0.30856 \mathrm{E}-00$ & $0.28929 \mathrm{E}-00$ & $0.42051 \mathrm{E}-01$ & $0.22786 \mathrm{E}-01$ \\
\hline 1.8 & $0.75351 \mathrm{E} \quad 00$ & $0.34224 \mathrm{E}-00$ & $0.26820 \mathrm{E}-00$ & $0.61095 \mathrm{E}-01$ & $0.26473 \mathrm{E}-01$ \\
\hline 2.0 & $0.65689 \mathrm{E} \quad 00$ & $0.36425 \mathrm{E}-00$ & $0.24721 \mathrm{E}-00$ & $0.83422 \mathrm{E}-01$ & $0.29700 \mathrm{E}-01$ \\
\hline 2.2 & $0.57682 \mathrm{E} \quad 00$ & $0.37379 \mathrm{E}-00$ & $0.22682 \mathrm{E}-00$ & $0.10804 \mathrm{E}-00$ & $0.32330 \mathrm{E}-01$ \\
\hline 2.4 & $0.51438 \mathrm{E} \quad 00$ & $0.37123 \mathrm{E}-00$ & $0.20747 \mathrm{E}-00$ & $0.13361 \mathrm{E}-00$ & $0.34271 \mathrm{E}-01$ \\
\hline 2.6 & $0.46907 \mathrm{E}-00$ & $0.35804 \mathrm{E}-00$ & $0.18949 \mathrm{E}-00$ & $0.15857 \mathrm{E}-00$ & $0.35478 \mathrm{E}-01$ \\
\hline 2.8 & $0.43902 \mathrm{E}-00$ & $0.33651 \mathrm{E}-00$ & $0.17311 \mathrm{E}-00$ & $0.18127 \mathrm{E}-00$ & $0.35950 \mathrm{E}-01$ \\
\hline 3.0 & $0.42139 \mathrm{E}-00$ & $0.30950 \mathrm{E}-00$ & $0.15847 \mathrm{E}-00$ & $0.20020 \mathrm{E}-00$ & $0.35734 \mathrm{E}-01$ \\
\hline 3.2 & $0.41273 \mathrm{E}-00$ & $0.28008 \mathrm{E}-00$ & $0.14560 \mathrm{E}-00$ & $0.21413 \mathrm{E}-00$ & $0.34908 \mathrm{E}-01$ \\
\hline 3.4 & $0.40944 \mathrm{E}-00$ & $0.25117 \mathrm{E}-00$ & $0.13445 \mathrm{E}-00$ & $0.22223 \mathrm{E}-00$ & $0.33579 \mathrm{E}-01$ \\
\hline 3.6 & $0.40814 \mathrm{E}-00$ & $0.22526 \mathrm{E}-00$ & $0.12493 \mathrm{E}-00$ & $0.22419 \mathrm{E}-00$ & $0.31872 \mathrm{E}-01$ \\
\hline 3.8 & $0.40602 \mathrm{E}-00$ & $0.20420 \mathrm{E}-00$ & $0.11685 \mathrm{E}-00$ & $0.22027 \mathrm{E}-00$ & $0.29917 \mathrm{E}-01$ \\
\hline 4.0 & $0.40106 \mathrm{E}-00$ & $0.18903 \mathrm{E}-00$ & $0.11003 \mathrm{E}-00$ & $0.21121 \mathrm{E}-00$ & $0.27842 \mathrm{E}-01$ \\
\hline 4.2 & $0.39217 \mathrm{E}-00$ & $0.17997 \mathrm{E}-00$ & $0.10427 \mathrm{E}-00$ & $0.19821 \mathrm{E}-00$ & $0.25762 \mathrm{E}-01$ \\
\hline 4.4 & $0.37910 \mathrm{E}-00$ & $0.17649 \mathrm{E}-00$ & $0.99342 \mathrm{E}-01$ & $0.18276 \mathrm{E}-00$ & $0.23774 \mathrm{E}-01$ \\
\hline 4.6 & $0.36243 \mathrm{E}-00$ & $0.17745 \mathrm{E}-00$ & $0.95069 \mathrm{E}-01$ & $0.16644 \mathrm{E}-00$ & $0.21952 \mathrm{E}-01$ \\
\hline 4.8 & $0.34331 \mathrm{E}-00$ & $0.18132 \mathrm{E}-00$ & $0.91279 \mathrm{E}-01$ & $0.15083 \mathrm{E}-00$ & $0.20345 \mathrm{E}-01$ \\
\hline 5.0 & $0.32321 \mathrm{E}-00$ & $0.18641 \mathrm{E}-00$ & $0.87835 \mathrm{E}-01$ & $0.13726 \mathrm{E}-00$ & $0.18977 \mathrm{E}-01$ \\
\hline 5.2 & $0.30371 \mathrm{E}-00$ & $0.19110 \mathrm{E}-00$ & $0.84634 \mathrm{E}-01$ & $0.12671 \mathrm{E}-00$ & $0.17849 \mathrm{E}-01$ \\
\hline 5.4 & $0.28619 \mathrm{E}-00$ & $0.19407 \mathrm{E}-00$ & $0.81603 \mathrm{E}-01$ & $0.11973 \mathrm{E}-00$ & $0.16943 \mathrm{E}-01$ \\
\hline 5.6 & $0.27172 \mathrm{E}-00$ & $0.19439 \mathrm{E}-00$ & $0.78700 \mathrm{E}-01$ & $0.11642 \mathrm{E}-00$ & $0.16230 \mathrm{E}-01$ \\
\hline 5.8 & $0.26088 \mathrm{E}-00$ & $0.19167 \mathrm{E}-00$ & $0.75904 \mathrm{E}-01$ & $0.11640 \mathrm{E}-00$ & $0.15670 \mathrm{E}-01$ \\
\hline 6.0 & $0.25373 \mathrm{E}-00$ & $0.18600 \mathrm{E}-00$ & $0.73214 \mathrm{E}-01$ & $0.11896 \mathrm{E}-00$ & $0.15220 \mathrm{E}-01$ \\
\hline 6.2 & $0.24986 \mathrm{E}-00$ & $0.17792 \mathrm{E}-00$ & $0.70639 \mathrm{E}-01$ & $0.12312 \mathrm{E}-00$ & $0.14839 \mathrm{E}-01$ \\
\hline 6.4 & $0.24848 \mathrm{E}-00$ & $0.16830 \mathrm{E}-00$ & $0.68193 \mathrm{E}-01$ & $0.12781 \mathrm{E}-00$ & $0.14491 \mathrm{E}-01$ \\
\hline 6.6 & $0.24857 \mathrm{E}-00$ & $0.15817 \mathrm{E}-00$ & $0.65890 \mathrm{E}-01$ & $0.13199 \mathrm{E}-00$ & $0.14150 \mathrm{E}-01$ \\
\hline 6.8 & $0.24902 \mathrm{E}-00$ & $0.14856 \mathrm{E}-00$ & $0.63741 \mathrm{E}-01$ & $0.13480 \mathrm{E}-00$ & $0.13795 \mathrm{E}-01$ \\
\hline 7.0 & $0.24883 \mathrm{E}-00$ & $0.14039 \mathrm{E}-00$ & $0.61751 \mathrm{E}-01$ & $0.13565 \mathrm{E}-00$ & $0.13419 \mathrm{E}-01$ \\
\hline 7.2 & $0.24722 \mathrm{E}-00$ & $0.13427 \mathrm{E}-00$ & $0.59916 \mathrm{E}-01$ & $0.13426 \mathrm{E}-00$ & $0.13019 \mathrm{E}-01$ \\
\hline 7.4 & $0.24372 \mathrm{E}-00$ & $0.13052 \mathrm{E}-00$ & $0.58227 \mathrm{E}-01$ & $0.13072 \mathrm{E}-00$ & $0.12602 \mathrm{E}-01$ \\
\hline 7.6 & $0.23824 \mathrm{E}-00$ & $0.12909 \mathrm{E}-00$ & $0.56669 \mathrm{E}-01$ & $0.12539 \mathrm{E}-00$ & $0.12177 \mathrm{E}-01$ \\
\hline 7.8 & $0.23100 \mathrm{E}-00$ & $0.12961 \mathrm{E}-00$ & $0.55225 \mathrm{E}-01$ & $0.11888 \mathrm{E}-00$ & $0.11756 \mathrm{E}-01$ \\
\hline 8.0 & $0.22254 \mathrm{E}-00$ & $0.13147 \mathrm{E}-00$ & $0.53873 \mathrm{E}-01$ & $0.11194 \mathrm{E}-00$ & $0.11352 \mathrm{E}-01$ \\
\hline 8.2 & $0.21354 \mathrm{E}-00$ & $0.13392 \mathrm{E}-00$ & $0.52596 \mathrm{E}-01$ & $0.10532 \mathrm{E}-00$ & $0.10976 \mathrm{E}-01$ \\
\hline 8.4 & $0.20476 \mathrm{E}-00$ & $0.13619 \mathrm{E}-00$ & $0.51377 \mathrm{E}-01$ & $0.99686 \mathrm{E}-01$ & $0.10634 \mathrm{E}-01$ \\
\hline 8.6 & $0.19689 \mathrm{E}-00$ & $0.13761 \mathrm{E}-00$ & $0.50201 \mathrm{E}-01$ & $0.95535 \mathrm{E}-01$ & $0.10329 \mathrm{E}-01$ \\
\hline 8.8 & $0.19047 \mathrm{E}-00$ & $0.13770 \mathrm{E}-00$ & $0.49062 \mathrm{E}-01$ & $0.93123 \mathrm{E}-01$ & $0.10063 \mathrm{E}-01$ \\
\hline 9.0 & $0.18580 \mathrm{E}-00$ & $0.13620 \mathrm{E}-00$ & $0.47953 \mathrm{E}-01$ & $0.92452 \mathrm{E}-01$ & $0.98310 \mathrm{E}-02$ \\
\hline 9.2 & $0.18293 \mathrm{E}-00$ & $0.13315 \mathrm{E}-00$ & $0.46874 \mathrm{E}-01$ & $0.93280 \mathrm{E}-01$ & $0.96272 \mathrm{E}-02$ \\
\hline 9.4 & $0.18165 \mathrm{E}-00$ & $0.12879 \mathrm{E}-00$ & $0.45827 \mathrm{E}-01$ & $0.95173 \mathrm{E}-01$ & $0.94440 \mathrm{E}-02$ \\
\hline 9.6 & $0.18154 \mathrm{E}-00$ & $0.12359 \mathrm{E}-00$ & $0.44815 \mathrm{E}-01$ & $0.97577 \mathrm{E}-01$ & $0.92736 \mathrm{E}-02$ \\
\hline 9.8 & $0.18204 \mathrm{E}-00$ & $0.11810 \mathrm{E}-00$ & $0.43843 \mathrm{E}-01$ & $0.99905 \mathrm{E}-01$ & $0.91088 \mathrm{E}-02$ \\
\hline 10.0 & $0.18257 \mathrm{E}-00$ & $0.11290 \mathrm{E}-00$ & $0.42913 \mathrm{E}-01$ & $0.10162 \mathrm{E}-00$ & $0.89440 \mathrm{E}-02$ \\
\hline$x$ & $I_{2}{ }^{2}(x)$ & $I_{3}{ }^{0}(x)$ & $I_{3}{ }^{1}(x)$ & $I_{3}{ }^{2}(x)$ & $I_{3}{ }^{3}(x)$ \\
\hline 0.0 & $0.24544 \mathrm{E}-01$ & & 0.00000 & 0.00000 & $0.68177 \mathrm{E}-03$ \\
\hline 0.2 & $0.24462 \mathrm{E}-01$ & $0.13576 \mathrm{E}-07$ & $0.40736 \mathrm{E}-06$ & $0.13584 \mathrm{E}-04$ & $0.68007 \mathrm{E}-03$ \\
\hline 0.4 & $0.24219 \mathrm{E}-01$ & $0.85752 \mathrm{E}-06$ & $0.64368 \mathrm{E}-05$ & $0.53730 \mathrm{E}-04$ & $0.67500 \mathrm{E}-03$ \\
\hline 0.6 & $0.23822 \mathrm{E}-01$ & $0.95559 \mathrm{E}-05$ & $0.31913 \mathrm{E}-04$ & $0.11865 \mathrm{E}-03$ & $0.66666 \mathrm{E}-03$ \\
\hline 0.8 & $0.23279 \mathrm{E}-01$ & $0.52065 \mathrm{E}-04$ & $0.97954 \mathrm{E}-04$ & $0.20546 \mathrm{E}-03$ & $0.65522 \mathrm{E}-03$ \\
\hline 1.0 & $0.22606 \mathrm{E}-01$ & $0.19089 \mathrm{E}-03$ & $0.23030 \mathrm{E}-03$ & $0.31038 \mathrm{E}-03$ & $0.64091 \mathrm{E}-03$ \\
\hline 1.2 & $0.21819 \mathrm{E}-01$ & $0.54296 \mathrm{E}-03$ & $0.45602 \mathrm{E}-03$ & $0.42889 \mathrm{E}-03$ & $0.62399 \mathrm{E}-03$ \\
\hline 1.4 & $0.20936 \mathrm{E}-01$ & $0.12924 \mathrm{E}-02$ & $0.79987 \mathrm{E}-03$ & $0.55601 \mathrm{E}-03$ & $0.60479 \mathrm{E}-03$ \\
\hline 1.6 & $0.19978 \mathrm{E}-01$ & $0.26935 \mathrm{E}-02$ & $0.12808 \mathrm{E}-02$ & $0.68654 \mathrm{E}-03$ & $0.58366 \mathrm{E}-03$ \\
\hline
\end{tabular}


TABLE 1 (Continued)

\begin{tabular}{|c|c|c|c|c|c|}
\hline$x$ & $I_{2}{ }^{2}(x)$ & $I_{3}{ }^{0}(x)$ & $I_{3}{ }^{1}(x)$ & $I_{3}{ }^{2}(x)$ & $I_{3}{ }^{3}(x)$ \\
\hline 1.8 & $0.18966 \mathrm{E}-01$ & $0.50600 \mathrm{E}-02$ & $0.19091 \mathrm{E}-02$ & $0.81535 \mathrm{E}--03$ & $0.56096 \mathrm{E}-03$ \\
\hline 2.0 & $0.17920 \mathrm{E}-01$ & $0.87397 \mathrm{E}-02$ & $0.26840 \mathrm{E}-02$ & $0.93759 \mathrm{E}-03$ & $0.53708 \mathrm{E}-03$ \\
\hline 2.2 & $0.16862 \mathrm{E}-01$ & $0.14075 \mathrm{E}-01$ & $0.35927 \mathrm{E}-02$ & $0.10490 \mathrm{E}-02$ & $0.51241 \mathrm{E}-03$ \\
\hline 2.4 & $0.15810 \mathrm{E}-01$ & $0.21356 \mathrm{E}-01$ & $0.46103 \mathrm{E}-02$ & $0.11459 \mathrm{E}-02$ & $0.48730 \mathrm{E}-03$ \\
\hline 2.6 & $0.14780 \mathrm{E}-01$ & $0.30763 \mathrm{E}-01$ & $0.57014 \mathrm{E}-02$ & $0.12257 \mathrm{E}-02$ & $0.46211 \mathrm{E}-03$ \\
\hline 2.8 & $0.13787 \mathrm{E}-01$ & $0.42323 \mathrm{E}-01$ & $0.68224 \mathrm{E}-02$ & $0.12864 \mathrm{E}-02$ & $0.43716 \mathrm{E}-03$ \\
\hline 3.0 & $0.12844 \mathrm{E}-01$ & $0.55872 \mathrm{E}-01$ & $0.79243 \mathrm{E}-02$ & $0.13274 \mathrm{E}-02$ & $0.41274 \mathrm{E}-03$ \\
\hline 3.2 & $0.11958 \mathrm{E}-01$ & $0.71032 \mathrm{E}-01$ & $0.89571 \mathrm{E}-02$ & $0.13487 \mathrm{E}-02$ & $0.38910 \mathrm{E}-03$ \\
\hline 3.4 & $0.11136 \mathrm{E}-01$ & $0.87224 \mathrm{E}-01$ & $0.98734 \mathrm{E}-02$ & $0.13511 \mathrm{E}-02$ & $0.36643 \mathrm{E}-03$ \\
\hline 3.6 & $0.10382 \mathrm{E}-01$ & $0.10369 \mathrm{E}-00$ & $0.10632 \mathrm{E}-01$ & $0.13363 \mathrm{E}-02$ & $0.34491 \mathrm{E}-03$ \\
\hline 3.8 & $0.96968 \mathrm{E}-02$ & $0.11956 \mathrm{E}-00$ & $0.11202 \mathrm{E}-01$ & $0.13066 \mathrm{E}-02$ & $0.32465 \mathrm{E}-03$ \\
\hline 4.0 & $0.90789 \mathrm{E}-02$ & $0.13393 \mathrm{E}-00$ & $0.11564 \mathrm{E}-01$ & $0.12645 \mathrm{E}-02$ & $0.30572 \mathrm{E}-03$ \\
\hline 4.2 & $0.85258 \mathrm{E}-02$ & $0.14593 \mathrm{E}-00$ & $0.11710 \mathrm{E}-01$ & $0.12129 \mathrm{E}-02$ & $0.28816 \mathrm{E}-03$ \\
\hline 4.4 & $0.80331 \mathrm{E}-02$ & $0.15482 \mathrm{E}-00$ & $0.11649 \mathrm{E}-01$ & $0.11545 \mathrm{E}-02$ & $0.27198 \mathrm{E}-03$ \\
\hline 4.6 & $0.75958 \mathrm{E}-02$ & $0.16012 \mathrm{E}-00$ & $0.11399 \mathrm{E}-01$ & $0.10923 \mathrm{E}-02$ & $0.25715 \mathrm{E}-03$ \\
\hline 4.8 & $0.72080 \mathrm{E}-02$ & $0.16157 \mathrm{E}-00$ & $0.10989 \mathrm{E}-01$ & $0.10286 \mathrm{E}-02$ & $0.24361 \mathrm{E}-03$ \\
\hline 5.0 & $0.68638 \mathrm{E}-02$ & $0.15927 \mathrm{E}-00$ & $0.10456 \mathrm{E}-01$ & $0.96574 \mathrm{E}-03$ & $0.23130 \mathrm{E}-03$ \\
\hline 5.2 & $0.65573 \mathrm{E}-02$ & $0.15361 \mathrm{E}-00$ & $0.98409 \mathrm{E}-02$ & $0.90540 \mathrm{E}-03$ & $0.22013 \mathrm{E}-03$ \\
\hline 5.4 & $0.62827 \mathrm{E}-02$ & $0.14525 \mathrm{E}-00$ & $0.91847 \mathrm{E}-02$ & $0.84894 \mathrm{E}-03$ & $0.21000 \mathrm{E}-03$ \\
\hline 5.6 & $0.60352 \mathrm{E}-02$ & $0.13508 \mathrm{E}-00$ & $0.85267 \mathrm{E}-02$ & $0.79725 \mathrm{E}-03$ & $0.20082 \mathrm{E}-03$ \\
\hline 5.8 & $0.58101 \mathrm{E}-02$ & $0.12411 \mathrm{E}-00$ & $0.79006 \mathrm{E}-02$ & $0.75082 \mathrm{E}-03$ & $0.19249 \mathrm{E}-03$ \\
\hline 6.0 & $0.56037 \mathrm{E}-02$ & $0.11334 \mathrm{E}-00$ & $0.73330 \mathrm{E}-02$ & $0.70975 \mathrm{E}-03$ & $0.18492 \mathrm{E}-03$ \\
\hline 6.2 & $0.54129 \mathrm{E}-02$ & $0.10371 \mathrm{E}-00$ & $0.68417 \mathrm{E}-02$ & $0.67386 \mathrm{E}-03$ & $0.17800 \mathrm{E}-03$ \\
\hline 6.4 & $0.52351 \mathrm{E}-02$ & $0.95967 \mathrm{E}-01$ & $0.64357 \mathrm{E}-02$ & $0.64272 \bar{E}-03$ & $0.17167 \mathrm{E}-03$ \\
\hline 6.6 & $0.50685 \mathrm{E}-02$ & $0.90596 \mathrm{E}-01$ & $0.61153 \mathrm{E}-02$ & $0.61577 \mathrm{E}-03$ & $0.16584 \mathrm{E}-03$ \\
\hline 6.8 & $0.49117 \mathrm{E}-02$ & $0.87792 \mathrm{E}-01$ & $0.58738 \mathrm{E}-02$ & $0.59235 \mathrm{E}-03$ & $0.16044 \mathrm{E}-03$ \\
\hline 7.0 & $0.47635 \mathrm{E}-02$ & $0.87441 \mathrm{E}-01$ & $0.56986 \mathrm{E}-02$ & $0.57180 \mathrm{E}-03$ & $0.15544 \mathrm{E}-03$ \\
\hline 7.2 & $0.46234 \mathrm{E}-02$ & $0.89158 \mathrm{E}-01$ & $0.55738 \mathrm{E}-02$ & $0.55347 \mathrm{E}-03$ & $0.15076 \mathrm{E}-03$ \\
\hline 7.4 & $0.44907 \mathrm{E}-02$ & $0.92342 \mathrm{E}-01$ & $0.54820 \mathrm{E}-02$ & $0.53682 \mathrm{E}-03$ & $0.14638 \mathrm{E}-03$ \\
\hline 7.6 & $0.43651 \mathrm{E}-02$ & $0.96263 \mathrm{E}-01$ & $0.54065 \mathrm{E}-02$ & $0.52136 \mathrm{E}-03$ & $0.14225 \mathrm{E}-03$ \\
\hline 7.8 & $0.42461 \mathrm{E}-02$ & $0.10016 \mathrm{E}-00$ & $0.53328 \mathrm{E}-02$ & $0.50677 \mathrm{E}-03$ & $0.13836 \mathrm{E}-03$ \\
\hline 8.0 & $0.41336 \mathrm{E}-02$ & $0.10332 \mathrm{E}-00$ & $0.52495 \mathrm{E}-02$ & $0.49278 \mathrm{E}-03$ & $0.13468 \mathrm{E}-03$ \\
\hline 8.2 & $0.40271 \mathrm{E}-02$ & $0.10520 \mathrm{E}-00$ & $0.51499 \mathrm{E}-02$ & $0.47926 \mathrm{E}-03$ & $0.13119 \mathrm{E}-03$ \\
\hline 8.4 & $0.39263 \mathrm{E}-02$ & $0.10546 \overline{\mathrm{E}}-00$ & $0.50310 \bar{E}-02$ & $0.46615 \mathrm{E}-03$ & $0.12788 \mathrm{E}-03$ \\
\hline 8.6 & $0.38308 \mathrm{E}-02$ & $0.10398 \mathrm{E}-00$ & $0.48938 \mathrm{E}-02$ & $0.45343 \mathrm{E}-03$ & $0.12473 \mathrm{E}-03$ \\
\hline 8.8 & $0.37403 \mathrm{E}-02$ & $0.10090 \mathrm{E}-00$ & $0.47424 \mathrm{E}-02$ & $0.44115 \mathrm{E}-03$ & $0.12174 \mathrm{E}-03$ \\
\hline 9.0 & $0.36543 \mathrm{E}-02$ & $0.96576 \mathrm{E}-01$ & $0.45830 \mathrm{E}-02$ & $0.42935 \mathrm{E}-03$ & $0.11889 \mathrm{E}-03$ \\
\hline 9.2 & $0.35724 \mathrm{E}-02$ & $0.91495 \mathrm{E}-01$ & $0.44227 \mathrm{E}-02$ & $0.41811 \mathrm{E}-03$ & $0.11617 \mathrm{E}-03$ \\
\hline 9.4 & $0.34943 \mathrm{E}-02$ & $0.86235 \mathrm{E}-01$ & $0.42685 \mathrm{E}-02$ & $0.40744 \mathrm{E}-03$ & $0.11357 \mathrm{E}-03$ \\
\hline 9.6 & $0.34196 \mathrm{E}-02$ & $0.81365 \mathrm{E}-01$ & $0.41261 \mathrm{E}-02$ & $0.39740 \mathrm{E}-03$ & $0.11110 \mathrm{E}-03$ \\
\hline 9.8 & $0.33481 \mathrm{E}-02$ & $0.77375 \mathrm{E}-01$ & $0.39999 \mathrm{E}-02$ & $0.38796 \mathrm{E}-03$ & $0.10873 \mathrm{E}-03$ \\
\hline 10.0 & $0.32795 \mathrm{E}-02$ & $0.74610 \mathrm{E}-01$ & $0.38920 \mathrm{E}-02$ & $0.37911 \mathrm{E}-03$ & $0.10647 \mathrm{E}-03$ \\
\hline
\end{tabular}

The contribution from the second interval in (3), with $m=0$, is

$$
\begin{aligned}
\int_{x^{1 / 2}}^{x} \frac{J_{n}^{2}(y)}{\sqrt{x^{2}-y^{2}}} d y & \cong \frac{2}{\pi} \int_{x^{1 / 2}}^{x} \frac{\cos ^{2}\left(y-\frac{\pi}{2} n-\frac{\pi}{4}\right)}{y \sqrt{x^{2}-y^{2}}} d y \\
& \cong \frac{1}{\pi} \int_{x^{1 / 2}}^{x}-\frac{d y}{y \sqrt{x^{2}-y^{2}}}=\frac{1}{\pi x} \log \left(x^{1 / 2}+\sqrt{x-1}\right) \\
& =\frac{1}{\pi x} \log 2 x^{1 / 2}+0\left(x^{-3 / 2}\right) .
\end{aligned}
$$

Thus, combining these results, we have the following asymptotic representations

$$
\begin{aligned}
I_{n}^{0}(x) & \sim \frac{1}{\pi x}\left[\log 4 x-\psi\left(n+\frac{1}{2}\right)\right] \\
I_{n}^{m}(x) & \sim \frac{1}{2 \sqrt{\pi} x} \frac{\Gamma(m)}{\Gamma\left(m+\frac{1}{2}\right)} \frac{\Gamma\left(n-m_{1}+\frac{1}{2}\right)}{\Gamma\left(n+m+\frac{1}{2}\right)} \quad(m=1,2, \cdots n) .
\end{aligned}
$$


The numerical evaluation of $I_{n}{ }^{m}(x)$ was performed on an IBM 7090 system, using two different methods. The first method consisted of evaluating the integral (1) by means of the Gauss-Mehler quadrature formula, whence

$$
I_{n}{ }^{m}(x) \cong \frac{\pi}{K} \sum_{k=1}^{K / 2} \frac{{J_{n}}^{2}\left(x \cos \frac{2 k-1}{2 K} \pi\right)}{\left(x \cos \frac{2 k-1}{2 K} \pi\right)^{2 m}}
$$

TABLE 2

The Integrals $I_{n+1 / 2}^{m+1 / 2}(x)$

\begin{tabular}{|c|c|c|c|c|c|}
\hline$x$ & $I_{3 / 2}^{1 / 2}(x)$ & $I_{3,2}^{1 / 2}(x)$ & $I_{3,2}^{3 / 2}(x)$ & $I_{5,2}^{1 / 2}(x)$ & $I_{5 ; 2}^{3 / 2}(x)$ \\
\hline 0.0 & $0.10000 \mathrm{E} \quad 01$ & 0.00000 & $0.11111 \mathrm{E}-00$ & 0.00000 & 0.00000 \\
\hline 0.2 & $0.99336 \mathrm{E} \quad 00$ & $0.22089 \mathrm{E}-02$ & $0.11067 \mathrm{E}-00$ & $0.26540 \mathrm{E}-05$ & $0.88509 \mathrm{E}-04$ \\
\hline 0.4 & $0.97376 \mathrm{E} \quad 00$ & $0.86780 \mathrm{E}-02$ & $0.10935 \mathrm{E}-00$ & $0.41861 \mathrm{E}-04$ & $0.34951 \mathrm{E}-03$ \\
\hline 0.6 & $0.94211 \mathrm{E} \quad 00$ & $0.18947 \bar{E}-01$ & $0.10720 \mathrm{E}-00$ & $0.20693 \mathrm{E}-03$ & $0.76972 \mathrm{E}-03$ \\
\hline 0.8 & $0.89991 \mathrm{E} \quad 00$ & $0.32294 \mathrm{E}-01$ & $0.10429 \mathrm{E}-00$ & $0.63246 \mathrm{E}-03$ & $0.13279 \mathrm{E}-02$ \\
\hline 1.0 & $0.84905 \mathrm{E} \quad 00$ & $0.47792 \mathrm{E}-01$ & $0.10069 \mathrm{E}-00$ & $0.14789 \mathrm{E}-02$ & $0.19963 \mathrm{E}-02$ \\
\hline 1.2 & $0.79176 \mathrm{E} \quad 00$ & $0.64391 \mathrm{E}-01$ & $0.96508 \mathrm{E}-01$ & $0.29087 \mathrm{E}-02$ & $0.27422 \mathrm{E}-02$ \\
\hline 1.4 & $0.73043 \mathrm{E} \quad 00$ & $0.80999 \mathrm{E}-01$ & $0.91868 \mathrm{E}-01$ & $0.50610 \mathrm{E}-02$ & $0.35299 \mathrm{E}-02$ \\
\hline 1.6 & $0.66746 \mathrm{E} \quad 00$ & $0.96569 \mathrm{E}-01$ & $0.86889 \mathrm{E}-01$ & $0.80283 \mathrm{E}-02$ & $0.43229 \mathrm{E}-02$ \\
\hline 1.8 & $0.60512 \mathrm{E} \quad 00$ & $0.11018 \mathrm{E}-00$ & $0.81696 \mathrm{E}-01$ & $0.11838 \mathrm{E}-01$ & $0.50863 \mathrm{E}-02$ \\
\hline 2.0 & $0.54539 \mathrm{E} \quad 00$ & $0.12111 \mathrm{E}-00$ & $0.76411 \mathrm{E}-01$ & $0.16439 \mathrm{E}-01$ & $0.57880 \mathrm{E}-02$ \\
\hline 2.2 & $0.48991 \mathrm{E}-00$ & $0.12886 \mathrm{E}-00$ & $0.71149 \mathrm{E}-01$ & $0.21702 \mathrm{E}-01$ & $0.64008 \mathrm{E}-02$ \\
\hline 2.4 & $0.43988 \mathrm{E}-00$ & $0.13319 \mathrm{E}-00$ & $0.66011 \mathrm{E}-01$ & $0.27422 \mathrm{E}-01$ & $0.69039 \mathrm{E}-02$ \\
\hline 2.6 & $0.39604 \mathrm{E}-00$ & $0.13415 \mathrm{E}-00$ & $0.61084 \mathrm{E}-01$ & $0.33336 \mathrm{E}-01$ & $0.72831 \mathrm{E}-02$ \\
\hline 2.8 & $0.35866 \mathrm{E}-00$ & $0.13200 \mathrm{E}-00$ & $0.56438 \mathrm{E}-01$ & $0.39143 \mathrm{E}-01$ & $0.75316 \mathrm{E}-02$ \\
\hline 3.0 & $0.32764 \mathrm{E}-00$ & $0.12720 \mathrm{E}-00$ & $0.52125 \mathrm{E}-01$ & $0.44531 \mathrm{E}-01$ & $0.76494 \mathrm{E}-02$ \\
\hline 3.2 & $0.30248 \mathrm{E}-00$ & $0.12036 \mathrm{E}-00$ & $0.48179 \mathrm{E}-01$ & $0.49206 \mathrm{E}-01$ & $0.76432 \mathrm{E}-02$ \\
\hline 3.4 & $0.28245 \mathrm{E}-00$ & $0.11216 \mathrm{E}-00$ & $0.44614 \mathrm{E}-01$ & $0.52919 \mathrm{E}-01$ & $0.75251 \mathrm{E}-02$ \\
\hline 3.6 & $0.26667 \mathrm{E}-00$ & $0.10329 \mathrm{E}-00$ & $0.41432 \mathrm{E}-01$ & $0.55489 \mathrm{E}-01$ & $0.73115 \mathrm{E}-02$ \\
\hline 3.8 & $0.25418 \mathrm{E}-00$ & $0.94407 \mathrm{E}-01$ & $0.38619 \mathrm{E}-01$ & $0.56818 \mathrm{E}-01$ & $0.70216 \mathrm{E}-02$ \\
\hline 4.0 & $0.24403 E-00$ & $0.86051 \mathrm{E}-01$ & $0.36152 \mathrm{E}-01$ & $0.56898 \mathrm{E}-01$ & $0.66760 \mathrm{E}-02$ \\
\hline 4.2 & $0.23538 \mathrm{E}-00$ & $0.78638 \mathrm{E}-01$ & $0.33999 \mathrm{E}-01$ & $0.55814 \mathrm{E}-01$ & $0.62954 \mathrm{E}-02$ \\
\hline 4.4 & $0.22755 \mathrm{E}-00$ & $0.72432 \mathrm{E}-01$ & $0.32126 \mathrm{E}-01$ & $0.53729 \mathrm{E}-01$ & $0.58992 \mathrm{E}-02$ \\
\hline 4.6 & $0.22001 \mathrm{E}-00$ & $0.67540 \mathrm{E}-01$ & $0.30492 \mathrm{E}-01$ & $0.50869 \mathrm{E}-01$ & $0.55047 \mathrm{E}-02$ \\
\hline 4.8 & $0.21245 \mathrm{E}-00$ & $0.63928 \mathrm{E}-01$ & $0.29062 \mathrm{E}-01$ & $0.47498 \mathrm{E}-01$ & $0.51259 \mathrm{E}-02$ \\
\hline 50 & $0.20471 \mathrm{E}-00$ & $0.61446 \mathrm{E}-01$ & $0.27798 \mathrm{E}-01$ & $0.43895 \mathrm{E}-01$ & $0.47738 \mathrm{E}-02$ \\
\hline 5.2 & $0.19679 \mathrm{E}-00$ & $0.59859 \mathrm{E}-01$ & $0.26668 \mathrm{E}-01$ & $0.40326 \mathrm{E}-01$ & $0.44556 \mathrm{E}-02$ \\
\hline 5.4 & $0.18880 \mathrm{E}-00$ & $0.58890 \mathrm{E}-01$ & $0.25647 \mathrm{E}-01$ & $0.37021 \mathrm{E}-01$ & $0.41750 \mathrm{E}-02$ \\
\hline 5.6 & $0.18092 \mathrm{E}-00$ & $0.58253 \mathrm{E}-01$ & $0.24710 \mathrm{E}-01$ & $0.34158 \mathrm{E}-01$ & $0.39329 \mathrm{E}-02$ \\
\hline 58 & $0.17334 \mathrm{E}-00$ & $0.57690 \mathrm{E}-01$ & $0.23841 \mathrm{E}-01$ & $0.31850 \mathrm{E}-01$ & $0.37274 \mathrm{E}-02$ \\
\hline 6.0 & $0.16626 \mathrm{E}-00$ & $0.56993 \mathrm{E}-01$ & $0.23027 \mathrm{E}-01$ & $0.30140 \mathrm{E}-01$ & $0.35548 \mathrm{E}-02$ \\
\hline 6.2 & $0.15982 \mathrm{E}-00$ & $0.56024 \mathrm{E}-01$ & $0.22258 \mathrm{E}-01$ & $0.29007 \mathrm{E}-01$ & $0.34102 \mathrm{E}-02$ \\
\hline 6.4 & $0.15409 \mathrm{E}-00$ & $0.54717 \mathrm{E}-01$ & $0.21530 \mathrm{E}-01$ & $0.28374 \mathrm{E}-01$ & $0.32879 \mathrm{E}-02$ \\
\hline 6.6 & $0.14909 \mathrm{E}-00$ & $0.53076 \mathrm{E}-01$ & $0.20840 \mathrm{E}-01$ & $0.28123 \mathrm{E}-01$ & $0.31824 \mathrm{E}-02$ \\
\hline 6.8 & $0.14478 \mathrm{E}-00$ & $0.51163 \mathrm{E}-01$ & $0.20185 \mathrm{E}-01$ & $0.28115 \mathrm{E}-01$ & $0.30886 \mathrm{E}-02$ \\
\hline 7.0 & $0.14104 \mathrm{E}-00$ & $0.49083 \mathrm{E}-01$ & $0.19565 \mathrm{E}-01$ & $0.28205 \mathrm{E}-01$ & $0.30022 \mathrm{E}-02$ \\
\hline 7.2 & $0.13776 \mathrm{E}-00$ & $0.46958 \mathrm{E}-01$ & $0.18980 \mathrm{E}-01$ & $0.28262 \mathrm{E}-01$ & $0.29199 \mathrm{E}-02$ \\
\hline 7.4 & $0.13477 \mathrm{E}-00$ & $0.44913 \mathrm{E}-01$ & $0.18429 \mathrm{E}-01$ & $0.28182 \mathrm{E}-01$ & $0.28395 \mathrm{E}-02$ \\
\hline 7.6 & $0.13194 \mathrm{E}-00$ & $0.43054 \mathrm{E}-01$ & $0.17912 \mathrm{E}-01$ & $0.27896 \mathrm{E}-01$ & $0.27598 \mathrm{E}-02$ \\
\hline 7.8 & $0.12914 \mathrm{E}-00$ & $0.41458 \mathrm{E}-01$ & $0.17426 \mathrm{E}-01$ & $0.27376 \mathrm{E}-01$ & $0.26804 \mathrm{E}-02$ \\
\hline 8.0 & $0.12631 \mathrm{E}-00$ & $0.40163 \mathrm{E}-01$ & $0.16970 \mathrm{E}-01$ & $0.26631 \mathrm{E}-01$ & $0.26016 \mathrm{E}-02$ \\
\hline 8.2 & $0.12339 \mathrm{E}-00$ & $0.39169 \mathrm{E}-01$ & $0.16541 \mathrm{E}-01$ & $0.25704 \mathrm{E}-01$ & $0.25242 \mathrm{E}-02$ \\
\hline 8.4 & $0.12038 \mathrm{E}-00$ & $0.38440 \mathrm{E}-01$ & $0.16136 \mathrm{E}-01$ & $0.24661 \mathrm{E}-01$ & $0.24491 \mathrm{E}-02$ \\
\hline 8.6 & $0.11731 \mathrm{E}-00$ & $0.37912 \mathrm{E}-01$ & $0.15754 \mathrm{E}-01$ & $0.23579 \mathrm{E}-01$ & $0.23774 \mathrm{E}-02$ \\
\hline 8.8 & $0.11425 \mathrm{E}-00$ & $0.37508 \mathrm{E}-01$ & $0.15391 \mathrm{E}-01$ & $0.22536 \mathrm{E}-01$ & $0.23097 \mathrm{E}-02$ \\
\hline 9.0 & $0.11126 \mathrm{E}-00$ & $0.37147 \mathrm{E}-01$ & $0.15044 \mathrm{E}-01$ & $0.21601 \mathrm{E}-01$ & $0.22467 \mathrm{E}-02$ \\
\hline 9.2 & $0.10840 \mathrm{E}-00$ & $0.36755 \mathrm{E}-01$ & $0.14712 \mathrm{E}-01$ & $0.20821 \mathrm{E}-01$ & $0.21885 \mathrm{E}-02$ \\
\hline 9.4 & $0.10573 \mathrm{E}-00$ & $0.36277 \mathrm{E}-01$ & $0.14394 \mathrm{E}-01$ & $0.20224 \mathrm{E}-01$ & $0.21351 \mathrm{E}-02$ \\
\hline 9.6 & $0.10330 \mathrm{E}-00$ & $0.35680 \mathrm{E}-01$ & $0.14087 \mathrm{E}-01$ & $0.19810 \mathrm{E}-01$ & $0.20861 \mathrm{E}-02$ \\
\hline 9.8 & $0.10111 \mathrm{E}-00$ & $0.34956 \mathrm{E}-01$ & $0.13792 \mathrm{E}-01$ & $0.19558 \mathrm{E}-01$ & $0.20409 \mathrm{E}-02$ \\
\hline 10.0 & $0.99155 \mathrm{E}-01$ & $0.34120 \mathrm{E}-01$ & $0.13508 \mathrm{E}-01$ & $0.19427 \mathrm{E}-01$ & $0.19987 \mathrm{E}-02$ \\
\hline
\end{tabular}


TABLE 2 (Continued)

\begin{tabular}{|c|c|c|c|c|c|}
\hline$x$ & $I_{5 / 2}^{5 / 2}(x)$ & $I_{7 / 2}^{1 / 2}(x)$ & $I_{7 / 2}^{3 / 2}(x)$ & $I_{7 / 2}^{5 / 2}(x)$ & $I_{7 / 2}^{7 / 2}(x)$ \\
\hline 0.0 & $0.44444 \mathrm{E}-02$ & 0.00000 & 0.00000 & 0.00000 & $0.90703 \mathrm{E}-04$ \\
\hline 0.2 & $0.44318 \mathrm{E}-02$ & $0.18070 \mathrm{E}-08$ & $0.54221 \mathrm{E}-07$ & $0.18080 \mathrm{E}-05$ & $0.90502 \mathrm{E}-04$ \\
\hline 0.4 & $0.43940 \mathrm{E}-02$ & $0.11431 \mathrm{E}-06$ & $0.85794 \mathrm{E}-06$ & $0.71601 \mathrm{E}-05$ & $0.89902 \mathrm{E}-04$ \\
\hline 0.6 & $0.43321 \mathrm{E}-02$ & $0.12769 \mathrm{E}-05$ & $0.42635 \mathrm{E}-05$ & $0.15844 \mathrm{E}-04$ & $0.88913 \mathrm{E}-04$ \\
\hline 0.8 & $0.42473 \mathrm{E}-02$ & $0.69813 \mathrm{E}-05$ & $0.13129 \mathrm{E}-04$ & $0.27518 \mathrm{E}-04$ & $0.87555 \mathrm{E}-04$ \\
\hline 1.0 & $0.41416 \mathrm{E}-02$ & $0.25711 \mathrm{E}-04$ & $0.31000 \mathrm{E}-04$ & $0.41727 \mathrm{E}-04$ & $0.85850 \mathrm{E}-04$ \\
\hline 1.2 & $0.40173 \mathrm{E}-02$ & $0.73533 \mathrm{E}-04$ & $0.61702 \mathrm{E}-04$ & $0.57926 \mathrm{E}-04$ & $0.83828 \mathrm{E}-04$ \\
\hline 1.4 & $0.38769 \mathrm{E}-02$ & $0.17618 \mathrm{E}-03$ & $0.10890 \mathrm{E}-03$ & $0.75507 \mathrm{E}-04$ & $0.81524 \mathrm{E}-04$ \\
\hline 1.6 & $0.37233 \mathrm{E}-02$ & $0.37001 \mathrm{E}-03$ & $0.17565 \mathrm{E}-03$ & $0.93825 \mathrm{E}-04$ & $0.78976 \mathrm{E}-04$ \\
\hline 1.8 & $0.35595 \mathrm{E}-02$ & $0.70127 \mathrm{E}-03$ & $0.26399 \mathrm{E}-03$ & $0.11223 \mathrm{E}-03$ & $0.76223 \mathrm{E}-04$ \\
\hline 2.0 & $0.33885 \mathrm{E}-02$ & $0.12235 \mathrm{E}-02$ & $0.37463 \mathrm{E}-03$ & $0.13010 \mathrm{E}-03$ & $0.73309 \mathrm{E}-04$ \\
\hline 2.2 & $0.32134 \mathrm{E}-02$ & $0.19928 \mathrm{E}-02$ & $0.50675 \mathrm{E}-03$ & $0.14686 \mathrm{E}-03$ & $0.70277 \mathrm{E}-04$ \\
\hline 2.4 & $0.30370 \mathrm{E}-02$ & $0.30620 \mathrm{E}-02$ & $0.65791 \mathrm{E}-03$ & $0.16200 \mathrm{E}-03$ & $0.67168 \mathrm{E}-04$ \\
\hline 2.6 & $0.28618 \mathrm{E}-02$ & $0.44733 \mathrm{E}-02$ & $0.82417 \mathrm{E}-03$ & $0.17512 \mathrm{E}-03$ & $0.64022 \mathrm{E}-04$ \\
\hline 2.8 & $0.26904 \mathrm{E}-02$ & $0.62509 \mathrm{E}-02$ & $0.10002 \mathrm{E}-02$ & $0.18590 \mathrm{E}-03$ & $0.60879 \mathrm{E}-04$ \\
\hline 3.0 & $0.25247 \mathrm{E}-02$ & $0.83948 \mathrm{E}-02$ & $0.11798 \mathrm{E}-02$ & $0.19416 \mathrm{E}-03$ & $0.57772 \mathrm{E}-04$ \\
\hline 3.2 & $0.23663 \mathrm{E}-02$ & $0.10876 \mathrm{E}-01$ & $0.13562 \mathrm{E}-02$ & $0.19981 \mathrm{E}-03$ & $0.54732 \mathrm{E}-04$ \\
\hline 3.4 & $0.22167 \mathrm{E}-02$ & $0.13635 \mathrm{E}-01$ & $0.15222 \mathrm{E}-02$ & $0.20287 \mathrm{E}-03$ & $0.51786 \mathrm{E}-04$ \\
\hline 3.6 & $0.20766 \mathrm{E}-02$ & $0.16581 \mathrm{E}-01$ & $0.16715 \mathrm{E}-02$ & $0.20346 \mathrm{E}-03$ & $0.48957 \mathrm{E}-04$ \\
\hline 3.8 & $0.19467 \mathrm{E}-02$ & $0.19600 \mathrm{E}-01$ & $0.17983 \mathrm{E}-02$ & $0.20177 \mathrm{E}-03$ & $0.46261 \mathrm{E}-04$ \\
\hline 4.0 & $0.18272 \mathrm{E}-02$ & $0.22557 \mathrm{E}-01$ & $0.18980 \mathrm{E}-02$ & $0.19809 \mathrm{E}-03$ & $0.43712 \mathrm{E}-04$ \\
\hline 4.2 & $0.17181 \mathrm{E}-02$ & $0.25312 \mathrm{E}-01$ & $0.19677 \mathrm{E}-02$ & $0.19271 \mathrm{E}-03$ & $0.41319 \mathrm{E}-04$ \\
\hline 4.4 & $0.16191 \mathrm{E}-02$ & $0.27726 \mathrm{E}-01$ & $0.20059 \mathrm{E}-02$ & $0.18598 \mathrm{E}-03$ & $0.39085 \mathrm{E}-04$ \\
\hline 4.6 & $0.15296 \mathrm{E}-02$ & $0.29677 \mathrm{E}-01$ & $0.20131 \mathrm{E}-02$ & $0.17824 \mathrm{E}-03$ & $0.37012 \mathrm{E}-04$ \\
\hline 4.8 & $0.14491 \mathrm{E}-02$ & $0.31072 \mathrm{E}-01$ & $0.19911 \mathrm{E}-02$ & $0.16985 \mathrm{E}-03$ & $0.35098 \mathrm{E}-04$ \\
\hline 5.0 & $0.13767 \mathrm{E}-02$ & $0.31849 \mathrm{E}-01$ & $0.19433 \mathrm{E}-02$ & $0.16111 \mathrm{E}-03$ & $0.33337 \mathrm{E}-04$ \\
\hline 5.2 & $0.13118 \mathrm{E}-02$ & $0.31993 \mathrm{E}-01$ & $0.18741 \mathrm{E}-02$ & $0.15230 \mathrm{E}-03$ & $0.31723 \mathrm{E}-04$ \\
\hline 5.4 & $0.12534 \mathrm{E}-02$ & $0.31528 \mathrm{E}-01$ & $0.17885 \mathrm{E}-02$ & $0.14367 \mathrm{E}-03$ & $0.30246 \mathrm{E}-04$ \\
\hline 5.6 & $0.12008 \mathrm{E}-02$ & $0.30523 \mathrm{E}-01$ & $0.16920 \mathrm{E}-02$ & $0.13541 \mathrm{E}-03$ & $0.28898 \mathrm{E}-04$ \\
\hline 5.8 & $0.11531 \mathrm{E}-02$ & $0.29082 \mathrm{E}-01$ & $0.15901 \mathrm{E}-02$ & $0.12766 \mathrm{E}-03$ & $0.27667 \mathrm{E}-04$ \\
\hline 6.0 & $0.11098 \mathrm{E}-02$ & $0.27332 \mathrm{E}-01$ & $0.14877 \mathrm{E}-02$ & $0.12051 \mathrm{E}-03$ & $0.26542 \mathrm{E}-04$ \\
\hline 6.2 & $0.10702 \mathrm{E}-02$ & $0.25416 \mathrm{E}-01$ & $0.13891 \mathrm{E}-02$ & $0.11401 \mathrm{E}-03$ & $0.25514 \mathrm{E}-04$ \\
\hline 6.4 & $0.10337 \mathrm{E}-02$ & $0.23479 \mathrm{E}-01$ & $0.12976 \mathrm{E}-02$ & $0.10818 \mathrm{E}-03$ & $0.24572 \mathrm{E}-04$ \\
\hline 6.6 & $0.99992 \mathrm{E}-03$ & $0.21651 \mathrm{E}-01$ & $0.12157 \mathrm{E}-02$ & $0.10299 \mathrm{E}-03$ & $0.23707 \mathrm{E}-04$ \\
\hline 6.8 & $0.96845 \mathrm{E}-03$ & $0.20041 \mathrm{E}-01$ & $0.11446 \mathrm{E}-02$ & $0.98399 \mathrm{E}-04$ & $0.22909 \mathrm{E}-04$ \\
\hline 7.0 & $0.93899 \mathrm{E}-03$ & $0.18726 \mathrm{E}-01$ & $0.10846 \mathrm{E}-02$ & $0.94346 \mathrm{E}-04$ & $0.22170 \mathrm{E}-04$ \\
\hline 7.2 & $0.91130 \mathrm{E}-03$ & $0.17747 \mathrm{E}-01$ & $0.10353 \mathrm{E}-02$ & $0.90760 \mathrm{E}-04$ & $0.21484 \mathrm{E}-04$ \\
\hline 7.4 & $0.88518 \mathrm{E}-03$ & $0.17108 \mathrm{E}-01$ & $0.99548 \mathrm{E}-03$ & $0.87570 \mathrm{E}-04$ & $0.20844 \mathrm{E}-04$ \\
\hline 7.6 & $0.86049 \mathrm{E}-03$ & $0.16777 \mathrm{E}-01$ & $0.96347 \mathrm{E}-03$ & $0.84703 \mathrm{E}-04$ & $0.20246 \mathrm{E}-04$ \\
\hline 7.8 & $0.83710 \mathrm{E}-03$ & $0.16697 \mathrm{E}-01$ & $0.93741 \mathrm{E}-03$ & $0.82097 \mathrm{E}-04$ & $0.19683 \mathrm{E}-04$ \\
\hline 8.0 & $0.81493 \mathrm{E}-03$ & $0.16789 \mathrm{E}-01$ & $0.91544 \mathrm{E}-03$ & $0.79697 \mathrm{E}-04$ & $0.19153 \mathrm{E}-04$ \\
\hline 8.2 & $0.79389 \mathrm{E}-03$ & $0.16969 \mathrm{E}-01$ & $0.89581 \mathrm{E}-03$ & $0.77458 \mathrm{E}-04$ & $0.18652 \mathrm{E}-04$ \\
\hline 8.4 & $0.77391 \mathrm{E}-03$ & $0.17151 \mathrm{E}-01$ & $0.87710 \mathrm{E}-03$ & $0.75345 \mathrm{E}-04$ & $0.18178 \mathrm{E}-04$ \\
\hline 8.6 & $0.75493 \mathrm{E}-03$ & $0.17263 \mathrm{E}-01$ & $0.85823 \mathrm{E}-03$ & $0.73335 \mathrm{E}-04$ & $0.17728 \mathrm{E}-04$ \\
\hline 8.8 & $0.73689 \mathrm{E}-03$ & $0.17249 \mathrm{E}-01$ & $0.83856 \mathrm{E}-03$ & $0.71411 \mathrm{E}-04$ & $0.17299 \mathrm{E}-04$ \\
\hline 9.0 & $0.71973 \mathrm{E}-03$ & $0.17079 \mathrm{E}-01$ & $0.81780 \mathrm{E}-03$ & $0.69565 \mathrm{E}-04$ & $0.16892 \mathrm{E}-04$ \\
\hline 9.2 & $0.70339 \mathrm{E}-03$ & $0.16746 \mathrm{E}-01$ & $0.79605 \mathrm{E}-03$ & $0.67793 \mathrm{E}-04$ & $0.16504 \mathrm{E}-04$ \\
\hline 9.4 & $0.68781 \mathrm{E}-03$ & $0.16265 \mathrm{E}-01$ & $0.77361 \mathrm{E}-03$ & $0.66094 \mathrm{E}-04$ & $0.16133 \mathrm{E}-04$ \\
\hline 9.6 & $0.67294 \mathrm{E}-03$ & $0.15673 \mathrm{E}-01$ & $0.75100 \mathrm{E}-03$ & $0.64469 \mathrm{E}-04$ & $0.15779 \mathrm{E}-04$ \\
\hline 9.8 & $0.65873 \mathrm{E}-03$ & $0.15016 \mathrm{E}-01$ & $0.72877 \mathrm{E}-03$ & $0.62919 \mathrm{E}-04$ & $0.15441 \mathrm{E}-04$ \\
\hline 10.0 & $0.64513 \mathrm{E}-03$ & $0.14349 \mathrm{E}-01$ & $0.70745 \mathrm{E}-03$ & $0.61446 \mathrm{E}-04$ & $0.15117 \mathrm{E}-04$ \\
\hline
\end{tabular}

where $K$ is an even integer. In the program $K=10$ was the initial value, this was iteratively doubled, and the resulting values of $I_{n}{ }^{m}(x)$ compared with the previous values until the difference in all computed values of $I_{n}{ }^{m}(x)$ was less than one part in 10,000 . In the range of the computations, $K=20$ was sufficient to give this accuracy. For the complete tabulation with integer values of $m$ and $n$, that is, for $x=.1(.1) 10.0, n=0(1) 9, m=0$ (1) $n$, this method required approximately 10 minutes of computing time on the IBM 7090. However, for $x \leqq .7$ this procedure broke down for large $m$, presumably owing to the very small values of the denominator. To overcome this difficulty, the series expression (2) was used, employing 
double-precision arithmetic to overcome the round-off error which occurred at large $x$. The series were truncated when the last term failed to change the sum by one part in $2^{-27}$. This method required approximately four minutes of 7090 time for the large table, and was thus more satisfactory in all respects. Comparison of the numerical results from these two methods for $x>.7$ showed complete agreement to $5 \mathrm{~S}$ with the exception of a very few scattered differences of a unit in the fifth figure. Since the use of the series seems likely to be the more accurate of the two methods, it is felt that the final results shown in Tables 1 and 2 can be considered as being accurate to 5 significant figures.

Hydromechanics Laboratory

David Taylor Model Basin

Washington 7, D. C.

1. G. N. Watson, A Treatise on the Theory of Bessel Functions, Cambridge 1958, paragraph 2.61, p. 32 . 